\title{
Gastric pentadecapeptide body protection compound BPC 157 and its role in accelerating musculoskeletal soft tissue healing
}

\author{
Daniel Gwyer ${ }^{1}$ - Nicholas M. Wragg ${ }^{2}$ - Samantha L. Wilson ${ }^{2}$
}

Received: 1 June 2018 / Accepted: 27 February 2019 / Published online: 27 March 2019

(C) The Author(s) 2019

\begin{abstract}
There is a current need for a therapy that can alleviate the social and economic burden that presents itself with debilitating and recurring musculoskeletal soft tissue injuries and disorders. Currently, several therapies are emerging and undergoing trials in animal models; these focus on the manipulation and administration of several growth factors implicated with healing. However, limitations include in vivo instability, reliance on biocompatible and robust carriers and restricted application procedures (local and direct). The aim of this paper is therefore to critically review the current literature surrounding the use of BPC 157, as a feasible therapy for healing and functional restoration of soft tissue damage, with a focus on tendon, ligament and skeletal muscle healing. Currently, all studies investigating BPC 157 have demonstrated consistently positive and prompt healing effects for various injury types, both traumatic and systemic and for a plethora of soft tissues. However, to date, the majority of studies have been performed on small rodent models and the efficacy of BPC 157 is yet to be confirmed in humans. Further, over the past two decades, only a handful of research groups have performed in-depth studies regarding this peptide. Despite this, it is apparent that BPC 157 has huge potential and following further development has promise as a therapy to conservatively treat or aid recovery in hypovascular and hypocellular soft tissues such as tendon and ligaments. Moreover, skeletal muscle injury models have suggested a beneficial effect not only for disturbances that occur as a result of direct trauma but also for systemic insults including hyperkalamia and hypermagnesia. Promisingly, there are few studies reporting any adverse reactions to the administration of BPC 157, although there is still a need to understand the precise healing mechanisms for this therapy to achieve clinical realisation.
\end{abstract}

Keywords BPC $157 \cdot$ Bepecin $\cdot$ Pentadecapeptide $\cdot$ Soft tissue injury $\cdot$ Corticosteroid interaction $\cdot$ Angiogenesis $\cdot$ Healing

\section{Introduction}

Some of the most frequently injured sites of the human body involve 'soft tissues' including skeletal muscles, tendons and ligaments. Musculoskeletal injuries of this nature can occur during both sporting and everyday activities. The majority of injury complaints, particularly those of a sporting nature, result from some form of incomplete or complete tear in the

Samantha L. Wilson

s.wilson2@lboro.ac.uk

1 National Centre for Sport and Exercise Medicine, School of Sport, Exercise and Health Sciences, Loughborough University, Epinal Way, Loughborough, Leicestershire LE11 3TU, UK

2 Centre for Biological Engineering, Wolfson School of Mechanical, Electrical and Manufacturing Engineering, Loughborough University, Epinal Way, Loughborough, Leicestershire LE11 3TU, UK fibres that comprise the structure of the functioning tissue or 'musculotendinous unit' (Sloan 2008). The healing capacity and timelines of recovery for these tissues vary significantly, depending on several factors. Some of these factors include the cellular composition and vascular nature of the tissue(s) in question.

The general healing process can be divided into three overlapping phases: (1) inflammatory phase (days 1-5), (2) repair/proliferative phase (days 5-14) and (3) remodelling phase (days 14-90+) (Hope and Saxby 2007). As an example, tendon tissue is composed primarily of tendon fibroblastic cells and extracellular matrix, which contains predominantly type I collagen, type III collagen and glycoproteins (Fukuta et al. 1998). Following the remodelling phase, a higher proportion of synthesised type I collagen, a corresponding decrease in cellularity, glycosaminoglycan content and type III collagen (Sharma and Maffulli 2005) can be observed. This change, coupled with the tissues already hypocellular and hypovascular nature (Liu et al. 2011), results 
in a 'slow' process of healing, particularly when addressing complete ruptures, which often require surgery. Even after 12 months post-injury or surgical intervention, the tissue still lacks the biomechanical and ultrastructural characteristics it had prior to the injury (Fox et al. 2017).

In the USA, figures from 2008 show that 33 million musculoskeletal injuries are reported per year, with $50 \%$ involving ligament and tendon injuries (James et al. 2008). Figures also show that an estimated 300,000 tendon and ligament repair surgeries are performed annually in the US alone (Pennisi 2002). Prevalence of injury is also mirrored in professional sporting populations, with the diagnostic grouping of 'most common injury' as skeletal muscle/tendon in nature, accounting for $45 \%$ of all diagnosed injuries (Brooks 2005). Thus, the need for a therapy that can alleviate the social and economic burden that presents itself with debilitating and recurring soft tissue injuries is apparent.

Currently, there are several therapies emerging and undergoing trials in animal models that focus on the manipulation and administration of several growth factors implicated in the healing process (Halper 2014; Park et al. 2017). However, limitations exist in the form of in vivo instability, the reliance on carriers that are biocompatible and robust and limited sites of application (local and direct) (Park et al. 2017). In comparison, an emerging treatment with potential therapeutic application, in the form of a pentadecapeptide known as BPC 157 (body protection compound), appears to be somewhat unrestricted by the limitations seen in previous therapies.

The aim of this paper is therefore to critically review the current literature surrounding the use of BPC 157, as a feasible therapy for soft tissue healing, with a focus on musculoskeletal tissues.

\section{Human gastric juice-derived protein: BPC 157}

The human gastric juice-derived protein labelled BPC 157, also known as Bepecin (Cox et al. 2017), PL 14736 and PL10 (Tkalčević et al. 2007) is a stable gastric pentadecapeptide that was first introduced and overviewed in the Journal of Physiology (Paris) by Sikirić et al. (1993). BPC 157 is a 15 amino acid fragment (Gly-Glu-Pro-Pro-Pro-Gly-Lys-Pro-AlaAsp-Asp-Ala-Gly-Leu-Val) that is often termed as 'synthetic'; although the compounds are 'natural', they are not known to exist in nature and are derived from another protein (Bódis et al. 1997). Since these early studies BPC 157 has shown significant promise in the healing of an abundance of tissues including but not limited to tendon (Staresinic et al. 2003; Krivic et al. 2006; Chang et al. 2010), ligaments (Lovric-Bencic et al. 2004; Cerovecki et al. 2010), skeletal muscle (Staresinic et al. 2006; Novinscak et al. 2008; Brcic et al. 2009) and bone (Šebečić et al. 1999). The following sections aim to explore and evaluate tissue-specific results, with an emphasis on musculoskeletal soft tissues.

\section{Tendon and ligament healing}

Due to a limited blood supply, the spontaneous healing of both tendons and ligaments is inherently poor. Thus, these soft tissues are highly prevalent in BPC 157 research. One of the earlier studies in literature investigated the applicability and therapeutic efficacy of BPC 157 via intraperitoneal administration, specifically the effect of BPC 157 on healing following the transection of the Achilles tendon in rats (Staresinic et al. 2003). It was revealed that, in comparison to the severely compromised healing observed in sham and control rats, the systemic delivery of BPC 157 significantly improved recovery measures (Staresinic et al. 2003). This was evidenced biochemically, micro- and macroscopically. Biomechanically, the healed tendons (over 14 days) showed an increased load to tendon failure and significantly higher functionality (Achilles functional Index-AFI). Microscopic analysis revealed a greater mononuclear count, less granulocytes, an increase in fibroblasts and superior formation of the reticulin and collagen fibres. Macroscopically, the defects treated with BPC 157 were smaller in size and depth and subsequently full tendon integrity was re-established (Staresinic et al. 2003). Similar results have also been shown in rat models investigating the healing of the medial collateral ligament (MCL) following surgical transection (Cerovecki et al. 2010). Treatment of BPC 157 was administered orally in drinking water, topically via a thin cream and via intraperitoneal administration over a 90-day period. This suggests that the peptide has a therapeutic benefit via a wide range of delivery mechanisms (Fig. 1).

A further study investigating tendon-to-bone healing following the transection and detachment of the Achilles tendon in rats went on to reveal that BPC 157 is also capable of promoting tendon-to-bone healing despite the presence of corticosteroids (Krivic et al. 2006). This is of significance since although corticosteroid use has long been and remains controversial for healing of tissues (Carrico et al. 1984; Walsh et al. 1995; Waters et al. 2000), it still remains a prevalent treatment choice clinically for soft tissue damage and inflammation. Thus, the fact that BPC 157 has the apparent ability to counteract corticosteroid aggravation (Krivic et al. 2006) lends itself to being applied alongside conventional treatments to improve both understanding and biomechanical outcomes.

Chang et al. (2010) attempted to elucidate the potential mechanism by which BPC 157 stimulates the outgrowth and proliferation of tendon fibroblasts. Results showed that BPC 157 significantly accelerated the outgrowth of tendon explants; furthermore, the in vitro migration and rate of spreading of tendon fibroblasts increased in a dose-dependent manner, which was attributed to the activation of the FAK-paxillin pathway. Although BPC 157 had no direct effect on the 


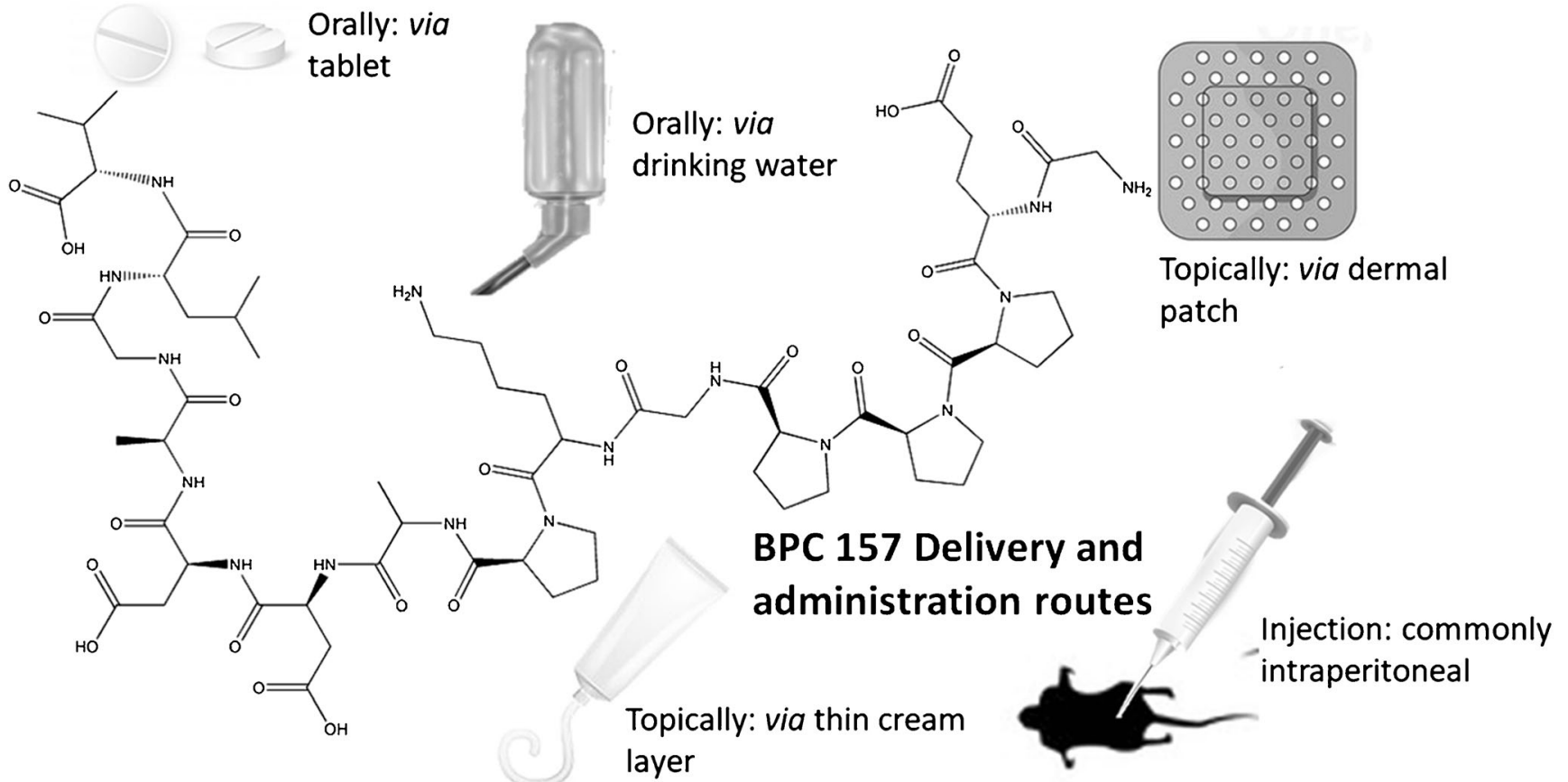

Fig. 1 Examples of successful administration mechanisms for the delivery of BPC 157; all routes, local and systemic, have been reported to have positive healing outcomes

proliferation of cultured tendon fibroblasts (Achilles), cell survival following $\mathrm{H}_{2} \mathrm{O}_{2}$ stress was significantly increased. The apparent lack of a direct effect was noted due to the in vitro environment not mimicking the inherent environment of a tendon in vivo.

\section{Skeletal muscle healing}

In addition to tendon and ligament healing, the positive therapeutic effect of BPC 157 has also been extended and applied to muscle injury models, both traumatic and systemic. Following the compete transection of the quadriceps muscle in rats, a traumatic definitive defect under normal healing conditions would not be compensated (Staresinic et al. 2006); it was reported that the systemic delivery of BPC 157 promoted healing. More importantly, the study demonstrated that this healing continued for a sustained period (72 days) whilst maintaining the functional restoration (Staresinic et al. 2006).

Novinscak et al. (2008) went on to compare the effectiveness of both systemic (intraperitoneal) and local treatment (as a thin cream layer) over a period of 14 days in crushed muscle (gastrocnemius muscle complex) in rats. BPC 157 significantly improved healing outcomes in both sites of treatment: macroscopically, microscopically and functionally, in addition to improving enzymatic activity (a decrease in muscle proteolysis) (Farges et al. 2002). The authors concluded that BPC 157 accelerated post- injury skeletal muscle healing in addition to restoring full muscle function that is similar to the finding in tendons reported by Staresinic et al. (2006).

Pevec et al. (2010), akin to the tendon-to-bone healing study of Krivic et al. (2006), proposed BPC 157 as an effective treatment that could improve muscle healing, when administered despite corticosteroid treatment, 6a-methylprednisolone, following gastrocnemius muscular injury. With a similar administrative procedure as previously outlined (topical and intraperitoneal), corticosteroid administration was shown to aggravate healing across all parameters. BPC 157 , in contrast, induced faster rates of healing and induced full functional restoration, which is also in agreement with the aforementioned studies on tendons (Krivic et al. 2006). When administered with corticosteroid, the restorative effects of BPC 157 were not significantly affected in functional, macroscopic or histological measures.

In addition to muscle injuries caused by direct trauma, there have been a number of studies that have indicated that BPC 157 may have the ability to recover systemic muscular disturbances in response to induced nervous, electrolyte disturbances and/or skeletal muscle wasting (Petek et al. 1999; Barisic et al. 2013; Klicek et al. 2013; Stambolija et al. 2016; Medvidovic-Grubisic et al. 2017; Kang et al. 2018). Since systemic muscle pain is attributed to infection, autoimmune conditions, illness or side effects from medication, they are considered to be more serious that stress or exerciserelated muscle injuries. 


\section{Prolonged therapeutic benefits}

In a study conducted by Hsieh et al. (2017), the authors aimed to explore the proangiogenic and therapeutic effect of BPC 157. Demonstrated by chick chorioallantoic membrane (CAM) and endothelial tube formation assays, both in vivo and in vitro, BPC 157 was shown to increase both vessel density and accelerate the recovery of blood flow in ischemic muscle, indicating a promotional effect on angiogenesis. Histological analysis was shown to confirm enhanced expression of vascular endothelial growth factor receptor 2 (VEGFR2) in rats treated with BPC 157. This was further confirmed in vitro using human vascular endothelial cells. BPC 157 was also shown to time-dependently activate the VEGFR2-Akt-eNOS signalling pathway. The study therefore demonstrates the pro-engiogenic effects of BPC 157 associated with VEGFR2 and VEGFR2-Akt-eNOS signalling.

It is worth noting that there does appear to be a trial administering BPC 157 (rectally administered) in human participants for the treatment and healing of acute to mild ulcerative colitis (Veljaca et al. 2002; Veljaca et al. 2003); however, details on the studies are limited and are not overly informative. Similarly, a pilot study in 2015 , a clinical trial (randomised) on 42 healthy human participants, receiving oral (tablet form) dosages of BPC 157 was carried out (Clinicaltrials.gov 2018): 0.25, 0.5, 1 and $2 \mu \mathrm{g} / \mathrm{kg}$. The details and results for this trial are still pending.

Although the mechanisms of action for BPC 157 are not yet fully understood, both in the currently reviewed and other surrounding literature, some light has been shed on a few potential systems involving nitric oxide (NO) (Sikiric et al. 2014), the FAK-paxillin pathway (Chang et al. 2010), VEGF (Hsieh et al. 2017) and the upregulation of growth hormone (receptor) (Chang et al. 2014).

\section{Comparison of published studies}

General strengths seen in all of the studies previously reported include the use of appropriate timelines for adequate stages of healing to take place; longer study periods are given for soft tissues that take longer to heal. All of the studies used both in vivo and some form of in vitro methods of data collection in order to observe different treatment effects, allowing for biomechanical, functional (use of AFI index and load to failure), macroscopic and microscopic histological analysis. All studies also reported the purity of BPC 157 in the methodologies (99\% high-pressure liquid chromatography (HPLC)).

Other strengths included allowing for appropriate time following administration of anaesthetic before biomechanical and functional testing to ensure that there was no interference between measures. The study conducted by Pevec et al. (2010) also compared effects following the combination of
BPC 157 and corticosteroids; this allows a further understanding of how well BPC 157 interacts with other agents and therefore its applicability in post-operative cases or following previous treatment. The studies specifically looking at soft tissue healing also spread the assessment of healing markers throughout the research period, involving data collection at various time points, for example, 1, 4, 7, 10 and 14 days throughout a 14-day study; this allows for a time-dependant relationship to be highlighted (Staresinic et al. 2003; Cerovecki et al. 2010; Pevec et al. 2010). Furthermore, different methods of administration - oral, intraperitoneal and topical - can be compared in order to establish a systemic effect. All studies used a range of dosages, from $10 \mu \mathrm{g}$, $10 \mathrm{ng}, 10 \mathrm{pg}$ and $0.1 \mathrm{pg}$ per different body masses $(\mathrm{kg} / \mathrm{bw})$ and volumes $(\mathrm{mL})$ BPC 157 in in vitro cultures (Staresinic et al. 2003; Hsieh et al. 2017). This allows for a dosedependent relationship to be established, as well as allowing the authors to gauge what dosages will affect micro vs macro level measurements.

Some of the most robust studies and methodologies were those that investigated the potential mechanisms surrounding the healing effects of BPC 157. For example, in the study by Novinscak et al. (2008), measures of enzyme activity were used (creatine kinase, lactate dehydrogenase, aspartate aminotransferase, alanine aminotransferase), allowing a gauge of trauma response (proteolysis) and therefore the ability to further understand the mechanisms of action. In the study conducted by Chang et al. (2010), both MTT and Transwell filter migration assays were used to establish cell proliferation of tendon fibroblasts, along with staining (FITC-phalloidin) and Western blot analysis to determine the protein expression and activation of FAK and paxillin. Finally, Hsieh et al. (2017) conducted chick chorioallantoic membrane (CAM), endothelial tube formation and enzyme-linked immunosorbent assays. One significant positive in this study is that they also used laser Doppler scanning (recovery of blood flow in the ischemic muscle) to indicate the promotion of angiogenesis alongside Western blot and real-time PCR for the expression of VEGFR2.

\section{Limitations of current studies}

When considering the limitations, not all studies used the same level of microscopic assessment, however, this is to be expected; the studies looking more specifically at mechanistic pathways of action will typically be focused on micro-analysis, whereas those looking at the level of tissue healing on a macro-scale will be focused on biomechanical and functional measures.

Despite the use of rodents and small mammals being commonplace in research, in particular for the development of novel therapeutic agents, caution still needs to be practiced 
when extrapolating research data to clinical applications. Notwithstanding the impressive results that have been published to date, there is still a requirement for successful human trials to be completed prior to clinical translation. Since there are obvious differences between rodent and human physiology, it cannot be ignored that this may have a significant impact on the efficacy and safety of (any) novel agents. However, it should also be noted that BPC 157 is a peptide derived from human gastric juices; therefore, some level of safety in human subjects can be assumed. However, this still cannot be taken as fact; thus, future studies should focus on elucidating as to whether the reported benefits of BPC 157 extend beyond research animals.

Another limitation of many of the cited studies was that although the majority of the authors gave justification for research (hypothesis specifically was not always outlined), they did not appear to critically evaluate their own methodologies. Finally, very few studies have touched upon any negatives associated with the use of BPC 157 (having no known toxicity level), as well as no obvious conflicts in literature-based ideas being seen; this could be due to a lack of understanding regarding its mechanisms, as well as the fact that over the past two decades, only a handful of studies have researched the peptide (many from the same laboratory or group).

Moving forwards, it would be beneficial for further research to progress to larger animal models. If more complex animal systems were observed in their stages of healing (larger animals with potentially more complex healing), this could provide more insight into the healing capabilities seen in larger mammals, such as humans. Another significant area of research would be into mechanisms of action, shedding more light on the supposed systemic healing capabilities of BPC 157. This could involve confirmation and further study of the mechanisms already suspected to be impacted in the healing process. A recently published review by Seiwerth et al. (2018) used the current understanding from tendon, ligament and bone and applied this to gastrointestinal tract healing, which could provide a pathway for understanding of the systemic healing capabilities for applications to other tissues. Contemporary studies looking at animal models with diseases that affect the vascular and endothelial systems have also provided new insight into how BPC 157 works (Radeljak et al. 2004; Sever et al. 2009; Duzel et al. 2017; Sikiric et al. 2018; Vukojević et al. 2018).

Despite the tumour-promoting effects of many growth factors and peptides, BPC 157 has been shown to inhibit and counteract increased expression of VEGF and subsequent signalling pathways (Radeljak et al. 2004; Sever et al. 2009) thus avoiding VEGF-tumorigenesis (Radeljak et al. 2004). Furthermore, BPC 157 inhibits the growth of several tumour lines and can counteract tumour cachexia (Kang et al. 2018).

Vukojević et al. (2018) and Duzel et al. (2017) recently submitted works utilising BPC 157 for the treatment of inferior caval vein ligatures and colitis in rats, respectively. ICV is used to represent Virchow's triad - hypercoagulability, heamodynamic changes and endothelial injury/dysfunctionand represents a subset of deep vein thrombosis (DVT). Application of BPC 157, which has innate endotheium protective properties, counteracted direct vein injuries, thrombosis and thrombocytopenia and prolonged bleeding (Vukojević et al. 2018). Furthermore, additional detrimenal consequences of ICV ligation were completely eliminated. When utilised as a protoype cryoprotective agent, BPC 157 has been seen to positively control blood vessel function in respsonse to performation and obstruction, thus re-establishing blood flow (Sikiric et al. 2018). Duzel et al. (2017) revealed that BPC 157 is fundamental to the tretament of colitis in rats; BPC 157 promotes the restoration of blood supply and vascular perfusion with spared mucosa. The integrity of the endothelium and oxidative damage could be restored and reversed respectively, which was linked to the increased expression and internalisation of VEGFR2.

Although not currently prescribed for human use, it is important for athletes to proceed with caution when looking at potential agents to prevent and treat injury. While not currently on the WADA list of banned substances (Wada-ama.org 2018), due to what some individuals would term as the synthetic nature of BPC 157, there may be issues associated with the use of this peptide, as seen by some sport organisations.

\section{Conclusion}

The reviewed research uses indicators of healing success in the form of in vitro, in vivo, macroscopic and microscopic level measures in various models. There appears to be no obvious conflict between studies surrounding the mechanisms of BPC 157. However, this may be due to BPC 157's clinical infancy and the limited number of published clinical trial articles exploring the use of the peptide. Nevertheless, all of the studies to date that have tested BPC 157 as a treatment therapy have demonstrated extremely positive healing effects for various injury types in a number of soft tissues. However, at present, studies are predominantly limited to small animal models (predominantly rodents) and the efficacy of BPC 157 is yet to be confirmed in human subjects. From the recent literature, it can be said that BPC 157 appears to avoid the majority of practical pitfalls for healing typically seen using peptidergic growth factors. Although further research is required in order to better understand its mechanisms and efficacy in practical settings, BPC 157 has the potential to be developed as a new therapy to conservatively treat or aid recovery following surgery in typically hypovascular and hypocellular soft tissues such as tendon and ligament tissue. 


\section{Compliance with ethical standards}

Conflict of interest The authors declare that they have no conflict of interest.

Open Access This article is distributed under the terms of the Creative Commons Attribution 4.0 International License (http:// creativecommons.org/licenses/by/4.0/), which permits unrestricted use, distribution and reproduction in any medium, provided you give appropriate credit to the original author(s) and the source, provide a link to the Creative Commons license and indicate if changes were made.

\section{References}

Barisic I, Balenovic D, Klicek R, Radic B, Nikitovic B, Drmic D, Udovicic M, Strinic D, Bardak D, Berkopic L, Djuzel V (2013) Mortal hyperkalemia disturbances in rats are NO-system related. The life saving effect of pentadecapeptide BPC 157. Regul Pept 181:50-66

Bódis B, Karádi O, Németh P, Dohoczky C, Kolega M, Mózsik G (1997) Evidence for direct cellular protective effect of PL-10 substances (synthesized parts of body protection compound, BPC) and their specificity to gastric mucosal cells. Life Sci 61:PL243-PL248

Brcic L, Brcic I, Staresinic M, Novinscak T, Sikiric P, Seiwerth S (2009) Modulatory effect of gastric pentadecapeptide BPC 157 on angiogenesis in muscle and tendon healing. J Physiol Pharmacol 60:191-196

Brooks J (2005) Epidemiology of injuries in English professional rugby union: part 2 training injuries. Br J Sports Med 39:767-775

Chang C, Tsai W, Hsu Y, Pang J (2014) Pentadecapeptide BPC 157 enhances the growth hormone receptor expression in tendon fibroblasts. Molecules 19:19066-19077

Carrico TJ, Mehrhof AI Jr, Cohen IK (1984) Biology of wound healing. Surg Clin N Am 64:721-733

Cerovecki T, Bojanic I, Brcic L, Radic B, Vukoja I, Seiwerth S, Sikiric P (2010) Pentadecapeptide BPC 157 (PL 14736) improves ligament healing in the rat. J Orthop Res 28:1155-1161

Chang C-H, Tsai W-C, Lin M-S, Hsu Y-H, Pang J-HS (2010) The promoting effect of pentadecapeptide BPC 157 on tendon healing involves tendon outgrowth, cell survival, and cell migration. J Appl Physiol 110:774-780

Clinicaltrials.gov. (2018) PCO-02 - Safety and pharmacokinetics trial Full Text View - ClinicalTrials.gov. NCT02637284 [online] Available at: https://clinicaltrials.gov/ct2/show/NCT02637284 [Accessed 20 Apr. 2018]

Cox H, Miller G, Eichner D (2017) Detection and in vitro metabolism of the confiscated peptides BPC 157 and MGF R23H. Drug Testing and Analysis 9:1490-1498

Duzel A, Vlainic J, Antunovic M, Malekinusic D, Vrdoljak B, Samara M, Gojkovic S, Krezic I, Vidovic T, Bilic Z, Knezevic M (2017) Stable gastric pentadecapeptide BPC 157 in the treatment of colitis and ischemia and reperfusion in rats: new insights. World $\mathrm{J}$ Gastroenterol 23:465-8488

Farges M, Balcerzak D, Fisher B, Attaix D, Béchet D, Ferrara M, Baracos $\mathrm{V}$ (2002) Increased muscle proteolysis after local trauma mainly reflects macrophage-associated lysosomal proteolysis. American Journal of Physiology-Endocrinology and Metabolism 282:E326E335

Fox G, Gabbe B, Richardson M, Oppy A, Page R, Edwards E, Hau R, Ekegren C (2017) Twelve-month outcomes following surgical repair of the Achilles tendon. J Sci Med Sport 20:e36-e37
Fukuta S, Oyama M, Kavalkovich K, Fu F, Niyibizi C (1998) Identification of types II, IX and $\mathrm{X}$ collagens at the insertion site of the bovine Achilles tendon. Matrix Biol 17:65-73

Halper J (2014) Advances in the use of growth factors for treatment of disorders of soft tissues. Adv Exp Med Biol 1:59-76

Hope M, Saxby T (2007) Tendon healing. Foot Ankle Clin 12:553-567

Hsieh MJ, Liu HT, Wang CN, Huang HY, Lin Y, Ko YS, Wang JS, Chang VH, Pang JH (2017) Therapeutic potential of pro-angiogenic BPC157 is associated with VEGFR2 activation and up-regulation. J Mol Med 95(3):323-333

James R, Kesturu G, Balian G, Chhabra A (2008) Tendon: biology, biomechanics, repair, growth factors, and evolving treatment options. The Journal of hand surgery 33:102-112

Kang EA, Han YM, An JM, Park YJ, Sikiric P, Kim DH, Kwon KA, Kim YJ, Yang D, Tchah H, Hahm KB (2018) BPC157 as potential agent rescuing from cancer cachexia. Curr Pharm Des 24:1947-1956

Kliček R, Kolenc D, Šuran J, Drmić D, Brčić L, Aralica G, Sever M, Holjevac J, Radić B, Turudić T, Kokot A (2013) Stable gastric pentadecapeptide BPC 157 heals cysteamine-colitis and coloncolon-anastomosis and counteracts cuprizone brain injuries and motor disability. J Physiol Pharmacol 64:597-612

Krivic A, Anic T, Seiwerth S, Huljev D, Sikiric P (2006) Achilles detachment in rat and stable gastric pentadecapeptide BPC 157: promoted tendon-to-bone healing and opposed corticosteroid aggravation. J Orthop Res 24:982-989

Liu C, Aschbacher-Smith L, Barthelery N, Dyment N, Butler D, Wylie C (2011) What we should know before using tissue engineering techniques to repair injured tendons: a developmental biology perspective. Tissue Eng B Rev 17:165-176

Lovric-Bencic M, Sikiric P, Hanzevacki JS, Seiwerth S, Rogic D, Kusec V, Aralica G, Konjevoda P, Batelja L, Blagaic AB (2004) Doxorubicinecongestive heart failure-increased big endothelin-1 plasma concentration: reversal by amlodipine, losartan, and gastric pentadecapeptide BPC157 in rat and mouse. J Pharmacol Sci 95:19-26

Medvidovic-Grubisic M, Stambolija V, Kolenc D, Katancic J, Murselovic T, Plestina-Borjan I, Strbe S, Drmic D, Barisic I, Sindic A, Seiwerth S (2017) Hypermagnesemia disturbances in rats, NO-related: pentadecapeptide BPC 157 abrogates, 1-NAME and 1-arginine worsen. Inflammopharmacology 25:439-449

Novinscak T, Brcic L, Staresinic M, Jukic I, Radic B, Pevec D, Mise S, Tomasovic S, Brcic I, Banic T (2008) Gastric pentadecapeptide BPC 157 as an effective therapy for muscle crush injury in the rat. Surg Today 38:716-725

Park J, Hwang S, Yoon I (2017) Advanced growth factor delivery systems in wound management and skin regeneration. Molecules 22: 1259

Pennisi E (2002) Tending Tender Tendons. Science 295:1011-1011

Pevec D, Novinscak T, Brcic L, Sipos K, Jukic I, Staresinic M, Mise S, Brcic I, Kolenc D, Klicek R, Banic T (2010) Impact of pentadecapeptide BPC 157 on muscle healing impaired by systemic corticosteroid application. Med Sci Monit 16(3):BR81-8

Sharma P, Maffulli N (2005) Basic biology of tendon injury and healing. Surgeon 3:309-316

Petek M, Sikiric P, Anic T, Buljat G, Šeparovic J, Stancic-Rokotov D, Seiwerth S, Grabarevic Z, Rucman R, Mikus D, Zoricic I (1999) Pentadecapeptide BPC 157 attenuates gastric lesions induced by alloxan in rats and mice. Journal of Physiology Paris 93:501-504

Radeljak S, Seiwerth S, Sikiric P (2004) BPC 157 inhibits cell growth and VEGF signalling via the MAPK kinase pathway in the human melanoma cell line. Melanoma Res 14:A14-A15

Seiwerth S, Rucman R, Turkovic B, Sever M, Klicek R, Radic B, Drmic D, Stupnisek M, Misic M, Vuletic LB, Pavlov KH (2018) BPC 157 and standard angiogenic growth factors. Gastrointestinal tract healing, lessons from tendon, ligament, muscle and bone healing. Curr Pharm Des 24:1972-1989 
Šebečić B, Nikolić V, Sikirić P, Seiwerth S, Šoša T, Patrlj L, Grabarević Ž, Ručman R, Petek M, Konjevoda P (1999) Osteogenic effect of a gastric pentadecapeptide, BPC 157 , on the healing of segmental bone defect in rabbits: a comparison with bone marrow and autologous cortical bone implantation. Bone 24:195-202

Sever M, Klicek R, Radic B, Brcic L, Zoricic I, Drmic D, Ivica M, Barisic I, Ilic S, Berkopic L, Blagaic AB (2009) Gastric pentadecapeptide BPC 157 and short bowel syndrome in rats. Dig Dis Sci 54:2070-2083

Sikiric P, Seiwerth S, Rucman R, Turkovic B, Rokotov D, Brcic L, Sever M, Klicek R, Radic B, Drmic D, Ilic S, Kolenc D, Aralica G, Stupnisek M, Suran J, Barisic I, Dzidic S, Vrcic H, Sebecic B (2014) Stable gastric pentadecapeptide BPC 157-NO-system relation. Curr Pharm Des 20:1126-1135

Sikirić P, Petek M, Ručman R, Seiwerth S, Grabarević Z, Rotkvić I, Turković B, Jagić V, Mildner B, Duvnjak M, Lang N, Danilović Z, Cviko A, Kolega M, Sallmani A, Djačić S, Bura M, Brkić T, Banić M, Dodig M, Corić V, Šimičević V, Veljaca M, Erceg D, Ježek D, Simunić-Banek L, Skroza N, Bulić K, Buljat G, Hanževački M, Orihovać V, Lučinger D, Culig J, Separović J, Marović A, Miše S, Suchanek E, Matoz W, Perović D, Gjurašin M, Mikulandra S, Derniković K, Cuk V, Karakas I (1993) A new gastric juice peptide, BPC. An overview of the stomach-stressorganoprotection hypothesis and beneficial effects of BPC. Journal of Physiology-Paris 87:313-327

Sikiric P, Rucman R, Turkovic B, Sever M, Klicek R, Radic B, Drmic D, Stupnisek M, Misic M, Vuletic LB, Pavlov KH (2018) Novel cytoprotective mediator, stable gastric pentadecapeptide BPC 157. Vascular recruitment and gastrointestinal tract healing. Curr Pharm Des 24:1990-2001

Sloan J (2008) Soft tissue injuries: introduction and basic principles. Emerg Med J 25:33-37

Stambolija V, Stambolija TP, Holjevac JK, Murselovic T, Radonic J, Duzel V, Duplancic B, Uzun S, Zivanovic-Posilovic G, Kolenc D, Drmic D (2016) BPC 157: the counteraction of succinylcholine, hyperkalemia, and arrhythmias. Eur J Pharmacol 781:83-91

Staresinic M, Petrovic I, Novinscak T, Jukic I, Pevec D, Suknaic S, Kokic N, Batelja L, Brcic L, Boban-Blagaic A (2006) Effective therapy of transected quadriceps muscle in rat: gastric pentadecapeptide BPC 157. J Orthop Res 24:1109-1117

Staresinic M, Sebecic B, Patrlj L, Jadrijevic S, Suknaic S, Perovic D, Aralica G, Zarkovic N, Borovic S, Srdjak M (2003) Gastric pentadecapeptide BPC 157 accelerates healing of transected rat Achilles tendon and in vitro stimulates tendocytes growth. J Orthop Res 21:976-983

Tkalčević V, Čužić S, Brajša K, Mildner B, Bokulić A, Šitum K, Perović D, Glojnarić I, Parnham M (2007) Enhancement by PL 14736 of granulation and collagen organization in healing wounds and the potential role of egr-1 expression. Eur J Pharmacol 570:212-221

Veljaca, M, Krnic, Z and Brajsa, K (2002) The development of PL 14736 for treatment of inflammatory bowel disease. IUPHAR-GI. Section symposium, Honolulu, Hawaii, 13:0-32

Veljaca M, Sladoljev P, Mildner B (2003) Tolerability and pharmacokinetics of PL 14736, a novel agent for treatment of ulcerative colitis, in healthy male volunteers. Gut 3:A309

Vukojević J, Siroglavić M, Kašnik K, Kralj T, Stanćić D, Kokot A, Kolarić D, Drmić D, Sever AZ, Barišić I, Šuran J (2018) Rat inferior caval vein (ICV) ligature and particular new insights with the stable gastric pentadecapeptide BPC 157. Vasc Pharmacol 106:54-66

Wada-ama.org. (2018) [online] Available at: https://www.wada-ama.org/sites/ default/files/prohibited_list_2018_en.pdf [Accessed 25 Apr. 2018]

Walsh W, Wiggins M, Fadale P, Ehrlich M (1995) Effects of a delayed steroid injection on ligament healing using a rabbit medial collateral ligament model. Biomaterials 16:905-910

Waters RV, Gamradt SC, Asnis P, Vickery BH, Avnur Z, Hill E, Bostrom M (2000) Systemic corticosteroids inhibit bone healing in a rabbit ulnar osteotomy model. Acta Orthop Scand 71:316-321

Publisher's note Springer Nature remains neutral with regard to jurisdictional claims in published maps and institutional affiliations. 